\title{
Fabricación y caracterización de nanoestructuras de Zno, y películas híbridas de ZnO/EOSIN-Y para la conversión de energía
}

Gronw and characterization of ZnO/EOSIN-Y hybrid thin films to photovoltaic conversion

Departamento de Física Aplicada, Universitat Politècnica de València. Camí de Vera s/n, 46022 , València, España.

\section{Resumen}

La viabilidad de utilizar la técnica de electrodeposición catódica para la preparación de nuevos materiales híbridos porosos ha sido probada en este artículo. Se prepararon películas finas híbridas de $\mathrm{ZnO}$ utilizando compuestos orgánicos como la Eosin-Y. La morfología y la porosidad de las películas híbridas de $\mathrm{ZnO}$ son altamente dependientes de la concentración de colorante que es utilizado. Fue observada una alta sensibilidad para las películas de $\mathrm{ZnO} /$ Eosin-Y mediante la excitación de los electrones de los niveles de

Doctor en Física Aplicada de la Universidad Politécnica de Valencia. Ingeniera Electrónica de la Universidad del Bosque en Bogotá Estudiante de Posdoctorado en la Pontificia Universidad Católica de Valparaíso en Chile, pertenece al grupo de investigación de electrónica de la misma universidad. E-mail:momoya@usbbog.edu.co

** Doctor en Física por la Universitat de València. Profesor de la Universidad Politécnica de Valencia. Director del título propio de la Universidad Politécnica de Valencia denominado Curso de Especialista Universitario en Instalaciones de Energía Solar Fototérmica y Fotovoltaica. Inició el montaje del Laboratorio de Semiconductores de la UPV. Desde entonces el profesor Marí ha liderado el grupo de investigación GOPS que se ha especializado en la preparación y caracterización de capas finas semiconductoras. E-mail: bmari@ fis.upv.es

*** Doctor en Química. Profesor titular de la Universidad Politécnica de Valencia en el Departamento de Física Aplicada y pertenece al Grupo de investigación de Optoelectrónica y Semiconductores (GOPS). E-mail: mmollar@fis.upv.es 
energía de Eosin-Y a través del ZnO. Igualmente, es posible controlar la porosidad de la superficie en las películas de $\mathrm{ZnO} /$ Eosin-Y.

\section{Palabras clave}

Electrodeposición, ZnO/eosin-Y; Películas delgadas cristalinas; Películas delgadas porosas, Mecanismo de crecimiento de nanoestructuras.

\section{Abstrac}

The feasibility of using the cathodic electrodeposition technique for preparing new porous hybrid materials has been tested in this paper. $\mathrm{ZnO}$ thin films were prepared using hybrid organic compounds such as Eosin-Y. The morphology and porosity of hybrid $\mathrm{ZnO}$ films are highly dependent on the concentration of dye to used. High sensitivity was observed for the films of $\mathrm{ZnO} /$ Eosin- $\mathrm{Y}$ by exciting electrons of energy levels of Eosin-Y through the $\mathrm{ZnO}$. It is also possible to control the porosity of the surface films of $\mathrm{ZnO} /$ Eosin-Y.

\section{Keywords}

Electrodeposition; ZnO/eosin-Y; Thin crystalline hybrid film; Hybrid film; Porous film; Growth mechanism.

\section{Introducción}

La posibilidad de electrodepositar materiales orgánicos e inorgánicos en un mismo proceso, es un novedoso método que ha llamado la atención para la fabricación de películas delgadas en aplicaciones de Células Solares Sensibilizadas con Colorantes (DSSC, por sus siglas en inglés, Day Sensitized Solar Cells) ${ }^{[1][6] .}$

Los compuestos inorgánicos de óxido de zinc $(\mathrm{ZnO})$ son un candidato prometedor para estas estructuras, debido a sus propiedades como la alta energía de enlace de excitación de $60 \mathrm{meV}^{[7]}$, un amplio ancho de banda prohibida (Gap) de 3.2 eV y una alta conductividad. Además, el $\mathrm{ZnO}$ puede ser depositado con gran facilidad utilizando la técnica de electrodeposición catódica (EDC) a bajas temperaturas $<100^{\circ} \mathrm{C}$ y obtener nanoestructuras híbridas depositadas con colorantes en un solo proceso, colorante como la Eosin-Y. En la actualidad, se ha reportado que las moléculas del colorante son fuertemente atraídas por la estructura de $\mathrm{ZnO}$ llenando los huecos mediante los grupos carboxilos ${ }^{[8]}$.

La implementación de estos materiales híbridos en la tecnología de dispositivos tecnológicos requiere de un control preciso en la estructura morfológica de las nanoestructuras. En este contexto, los procedimientos electroquímicos son adecuados desde el punto de vista de crecimiento de diferentes morfologías con el manejo de diferentes parámetros como el tipo de solvente en el electrolito y la concentración adecuada de los compuestos que conforman la disolución. 
Recientemente, cálculos teóricos usando la teoría de densidad funcional proponen un mecanismo de dos electrones para explicar la inyección del electrón desde un estado excitado del Eosin-Y a la banda de valencia del $\mathrm{ZnO}{ }^{[9]}$. Teniendo en cuenta esta investigación, la directa excitación de la banda HOMO, Orbital Molecular Ocupado más Alto (Highest Occuped Molecular Orbital) $\rightarrow$ LUMO, Orbital Molecular No-ocupado más Bajo (Lowest Unoccupied Molecular Orbital) en la Eosin-Y, no es suficiente para producir una inyección del electrón dentro del semiconductor, debido a que los estados HOMO y LUMO del colorante se encuentran dentro de la banda gap del $\mathrm{ZnO}$. Cuando el electrón se localiza en un nivel LUMO+1 puede ser inyectado en el $\mathrm{ZnO}$.

En este artículo, reportamos la morfología, estructura cristalina y propiedades ópticas de las películas de $\mathrm{ZnO}$ y de las películas delgadas híbridas de $\mathrm{ZnO}$ /Eosin-Y realizadas por electrodeposición catódica bajo diferentes condiciones de crecimiento. Así mismo, es reportado el comportamiento fotoelectroquímico para las películas híbridas.

\section{Detalles experimentales}

\section{A. Detalles experimentales}

La EDC de ZnO fue llevada a cabo en una celda electroquímica de tres-electrodos compuesta por un cátodo, que consiste en un sustrato de vidrio conductor, óxido de indio dopado con estaño (ITO) con una resistencia de $\approx 10 \Omega / \square$, un contraelectrodo de platino (Pt) y un electrodo de referencia $\mathrm{Ag} / \mathrm{AgCl}$ (+222 mV Vs electrodo normal de hidrógeno). Fueron preparados dos baños diferentes de disolución de electrolito. El primer baño contenía una mezcla de $0.1 \mathrm{M}$ de $\mathrm{KCl}$ (Merck) y $5 \times 10^{-3} \mathrm{M}$ de $\mathrm{ZnCl}_{2}$ (Merck) y 1 × $10^{-4} \mathrm{M}$ de Eosin-Y (Sigma Aldrich, España). Mientras que el segundo contenía una concentración diferente de colorante de $4 \times 10^{-4} \mathrm{M}$. El baño de electrolito fue purgado por $\mathrm{O}_{2}$ y agitado durante todo el proceso mediante un agitador magnético con el fin de facilitar la difusión del oxígeno. Antes del proceso de electrodeposición los sustratos de vidrio conductor ITO, fueron puestos 15 minutos en el ultrasonido en una disolución de acetona, seguido de 15 minutos en etanol y después fueron lavados con agua destilada y secados con un secador convencional. El tiempo total de la deposición de las películas híbridas de $\mathrm{ZnO} /$ Eosin-Y aproximadamente es de 350-600 s. La deposición potenciostática fue llevada a cabo a un potencial de $-0.9 \mathrm{~V}$ para un sustrato de una área de $1 \mathrm{~cm}^{2}$, utilizando un potenciostato/ galvanostato Autolab. El proceso de electrodeposición fue llevado a cabo a una temperatura de $80{ }^{\circ} \mathrm{C}$ controlada por un termostato. Después de la deposición, las películas de $\mathrm{ZnO} /$ Eosin-Y fueron lavadas con agua destilada y secadas.

\section{B. Caracterización}

La morfología de las películas de ZnO/Eosin-Y fue estudiada mediante el Microscopio Electrónico de Barrido (SEM) de marca JEOL-JSM6300 operado a 10 $k V$. Para la caracterización estructural fue utilizado un difractómetro de rayos-X (XRD) de alta resonancia de marca Rigacu Ultima IV en modo $\theta-2 \theta$ con un anticátodo de cobre (CuKa, $1.54 \AA$ ). Las medidas de transmitancia fueron realizadas mediante 
el instrumento Ocean Optics DT-MINI-2-GS con una lámpara de deuteriohalógeno acoplado a un detector espectrómetro para el rango de luz UV-Vis. Para determinar la superficie de topográfica se utilizó un Microscopio de Fuerza Atómica (AFM) de referencia Multimode Veeco en modo "tapping» usando una punta de silicona. El estudio futoelectroquímico fue llevado a cabo en una celda convencional de vidrio de tres-electrodos, que consiste en un electrodo de trabajo (la película delgada depositada de $\mathrm{ZnO} /$ Eoisn-Y), un contraelectrodo de Pt y un electrodo de referencia de $\mathrm{Ag} / \mathrm{AgCl}$, todos introducidos en una disolución de $0.1 \mathrm{M}$ de $\mathrm{KCl}$. Las medidas de fotocorriente fueron monitoreadas usando el potenciostato/galvanostato Autolab. El tiempo de iluminación del electrolito fue controlado usando un obturador mecánico/automático con un tiempo ajustable de iluminación de $10 \mathrm{~s}$ y a un potencial de $0.05 \mathrm{~V}$.

\section{Resultados}

\section{A. Caracterización de la morfología mediante SEM}

La figura 1 muestra las micrografías de las películas de $\mathrm{ZnO}$ puro y las películas híbridas de $\mathrm{ZnO} /$ Eosin-Y todas depositadas en agua a una temperatura de $80{ }^{\circ} \mathrm{C}$ y un potencial de $-0.9 \mathrm{~V}$. La figura 1 a) exhibe una estructura de columnas de $\mathrm{ZnO}$ perfectamente definidas en su estructura hexagonal, principalmente orientadas a lo largo del eje-c. La morfología de las películas híbridas de $\mathrm{ZnO} /$ Eosin-Y muestra nanopartículas conectadas de forma aleatoria a lo largo de la superficie del sustrato formando una estructura altamente porosa, esto puede conseguirse cuando se utiliza una concentración de $\left[\right.$ Eosin-Y] $=1 \times 10^{-4} \mathrm{M}$, como se ve en la figura $1 \mathrm{~b}$ ). Mientras, que cuando la concentración de [Eosin-Y] incrementa a $4 \times 10^{-4} \mathrm{M}$ se obtiene una estructura completamente diferente. La figura 1 c) muestra para este caso que los granos tienden a incrementar su tamaño aproximadamente dos veces $(\approx 600 \mathrm{~nm})$ respecto a las películas depositadas con $1 \times 10^{-4} \mathrm{M}(\approx 300 \mathrm{~nm}) \mathrm{de}$ Eosin-Y. También, se observa una estructura más lisa y con grietas sobre toda la superficie del sustrato.

\section{B. Caracterización de la nanoestructura de las películas de Zno}

La superficie topográfica de las nanoestructuras híbridas de $\mathrm{ZnO} /$ Eosin-Y y las nanoestructuras de $\mathrm{ZnO}$ puro son mostradas en las figura 2 a) y figura $2 b$ ) respectivamente. Las películas híbridas de $\mathrm{ZnO} /$ Eosin-Y muestran dominios del mismo tamaño formados por una morfología mesoporosa con poros abiertos. También, se puede observar la formación de grupos de Eosin- $-\mathrm{Y}^{4+} / \mathrm{Zn}^{2+}$, en donde las orillas de las partículas fueron indistintos de una morfología granular y compacta. Por otro lado, el $\mathrm{ZnO}$ puro está compuesto por largos cristales que cubren toda la superficie del sustrato. La conductividad es suficientemente alta y permite la formación de un proceso redox. A su vez, esta estructura es apta para el uso de colorantes, debido a los espacios que se crean entre las columnas haciendo que las moléculas de colorante se intercalen en la estructura. 

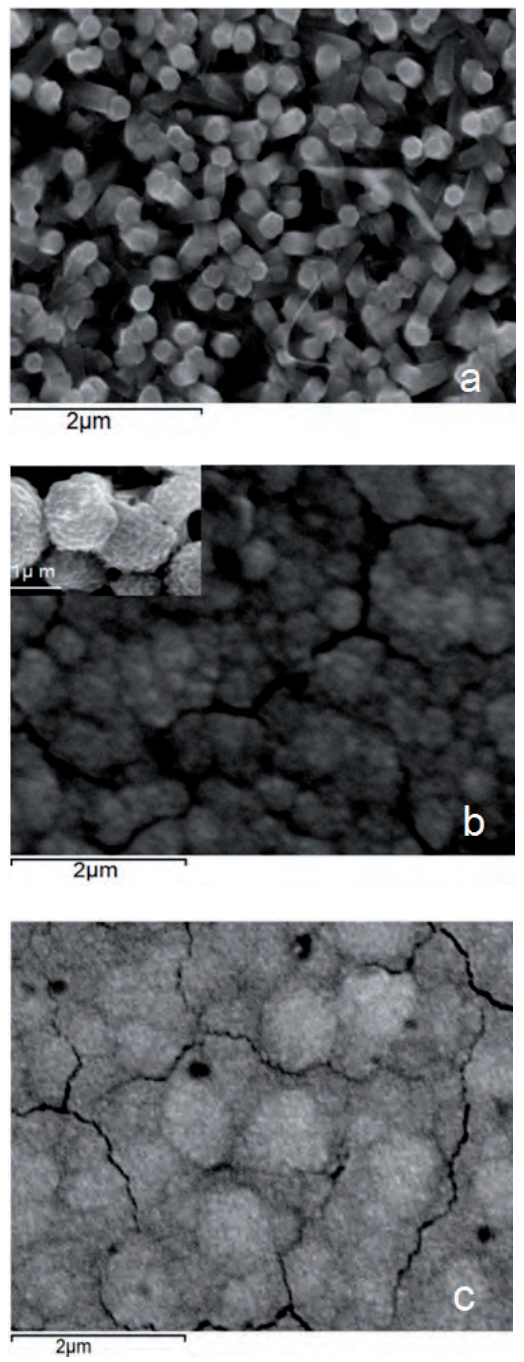

Figura 1. Micrografías SEM de (a) $\mathrm{ZnO}$ puro crecidas a $\mathrm{T}=80^{\circ} \mathrm{C}, \mathrm{V}=-0.9 \mathrm{~V}$, (b) $\mathrm{ZnO} /$ Eosin-Y películas crecidas a $\mathrm{T}=80^{\circ} \mathrm{C}, \mathrm{V}=-0.9 \mathrm{~V}$ con una concentración de Eosin- $\mathrm{Y} 1$ x 10-4 M y (c) ZnO/Eosin-Y películas crecidas a $\mathrm{T}=80^{\circ} \mathrm{C}, \mathrm{V}=-0.9 \mathrm{~V}$ con una concentración de Eosin- $\mathrm{Y} 4 \times 10-4 \mathrm{M}$

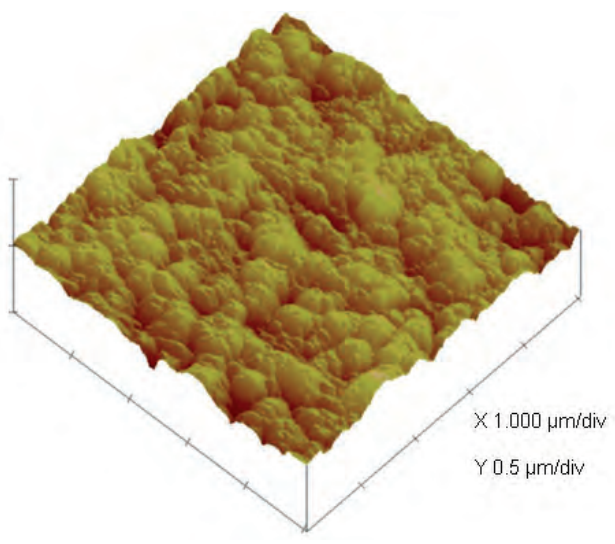

a) $\mathrm{ZnO} /$ Eosin-Y



b) ZnO puro

Figura 2. Imágenes de AFM. a) Películas híbridas de $\mathrm{ZnO} /$ Eosin-Y y b) ZnO puro 


\section{Propiedades ópticas}

Las propiedades ópticas de las películas híbridas fueron estudiadas por espectroscopia de absorción UV-VIS y mostradas en la figura 3. El espectro de absorbancia de una disolución pura de Eosin-Y en agua es mostrada en la figura 3 a). La Eosin-Y muestra una amplia banda de absorción centrada alrededor de $516 \mathrm{~nm}$. El espectro de absorbancia de dos películas híbridas preparadas con diferente concentración [Eosin-Y] $=1 \times 10^{-4} \mathrm{M}$ y $\left[\right.$ Eosin-Y] $=4 \times 10^{-4} \mathrm{M}$ es mostrado en la figura $3 \mathrm{~b}$ ). Para este estudio solo la contribución del colorante se tuvo en cuenta en los espectros de absorbancia y de transmitancia. También, se pudo confirmar que la tendencia de la absorbancia incrementa a medida que aumenta la concentración del colorante en la película híbrida, este efecto puede ser observado a simple vista en las películas híbridas cuando se obtiene un color más intenso en las películas de mayor concentración. De acuerdo con la ley de Lambert-Beer, la cantidad de colorante cargado dentro de la película híbrida es proporcional a la intensidad de las bandas de absorción. Sin embargo, para este caso la intensidad de los espectros de absorción representa la verdadera concentración del colorante que puede absorber la radiación y no la cantidad total del colorante en la película.
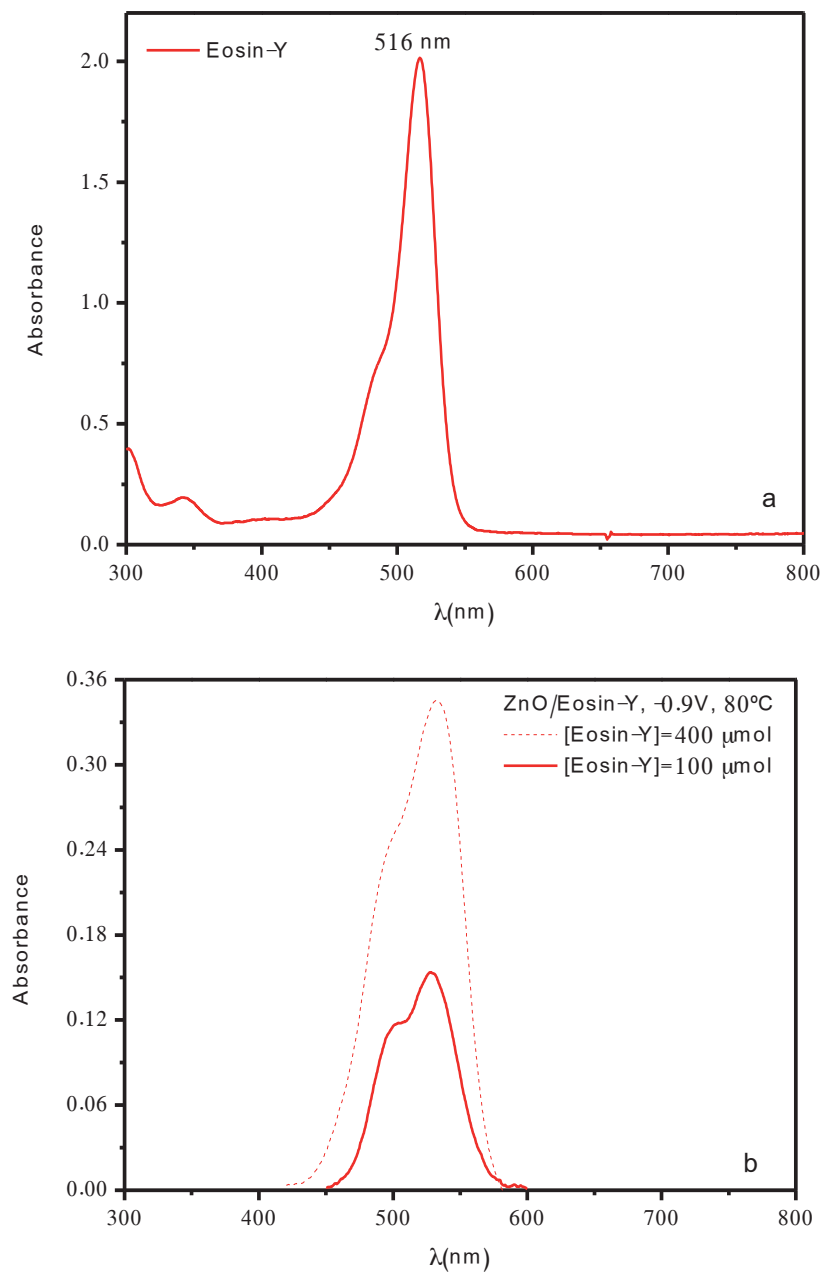

Figura 3. Espectros de Absorción de las películas híbridas de $\mathrm{ZnO} /$ Eosin-Y con dos diferentes concentraciones. [Eosin-Y] $=1$ x 10-4M y [Eosin-Y] $=4$ × 10-4M 


\section{Difracción de RXD}

En la figura 4 se puede observar el efecto que tiene el colorante sobre los patrones de RXD de la estructura de $\mathrm{ZnO}$ en su forma wurtzita y de las películas híbridas de $\mathrm{ZnO} /$ Eosin-Y. Tanto el ZnO como las películas híbridas de ZnO/Eosin-Y muestran una alta orientación preferencial en la dirección (002). Como se sabe, cada estructura tiene esta orientación preferencial con el eje-c paralelo al plano del sustrato, concuerda con las columnas verticales hexagonales, que son mostradas en la figura 1 a). Las estructuras de las películas híbridas de $\mathrm{ZnO} /$ Eosin-Y, revelan una mejora visible en comparación con las películas de puro $\mathrm{ZnO}$. La mejora del dominio cristalino puede ser confirmado mediante la estimación del tamaño del grano utilizando la fórmula de Scherrer, esto solo es factible para estructuras con un tamaño de grano $<100 \mathrm{~nm}$, teniendo en cuenta el Ancho Máximo de Pico Medio, (FWHM, por sus siglas en inglés, Full width at Half Máximum) de cada espectro [10] [11] para cada uno de los picos de interés. Por lo tanto, el tamaño de grano de los cristales para las películas de ZnO/Eosin-Y está alrededor de $30 \mathrm{~nm}$. En conclusión, la orientación cristalográfica de las películas de ZnO puro, permanece sin cambios debido a los colorantes, sin embargo, fue comprobado que al utilizar colorante en las películas de $\mathrm{ZnO}$ para la formación de películas híbridas de $\mathrm{ZnO} /$ Eosin-Y se consigue una mejora en la intensidad del pico relativo. La intensidad de los picos relativos de los espectros de RXD son mostrados en la tabla 1, en donde se puede confirmar la orientación preferencial (002) de los cristales de las películas de ZnO puro y de las películas híbridas de $\mathrm{ZnO} /$ Eosin-Y.



Figura 4. Espectros $\mathrm{XRD}$ del $\mathrm{ZnO}$ puro y de las películas híbridas de $\mathrm{ZnO} /$ Eosin-Y con [Eosin-Y] $=1 \times 10-4 \mathrm{M}$

\begin{tabular}{|c|c|c|}
\hline $\mathrm{d}(\mathrm{hkl})$ & Zn0 & Eosin-Y \\
\hline \hline$(002)$ & 1 & 1 \\
\hline \hline$(101)$ & 0.10 & 0.002 \\
\hline \hline$(100)$ & 0.05 & 0.007 \\
\hline
\end{tabular}

Tabla 1. Intensidad relativa de los picos de los espectros de RXD de las películas de $\mathrm{ZnO}$ y Eosin-Y 


\section{E. Análisis de la respuesta de fotocorriente}

La figura 5 muestra la respuesta de fotocorriente generada en las películas híbridas de $\mathrm{ZnO} /$ Eoisn-Y después de iluminación con una luz monocromática. La máxima eficiencia hasta el momento para las películas híbridas de ZnO/Eoisn-Y aplicadas en DSSC ha sido $2.3 \%{ }^{[12]}$ teniendo en cuenta la similitud de los niveles de energía que existen entre el ZnO y el Eosin-Y. Se pude apreciar, un exceso a la respuesta de fotocorriente por la incidencia de luz que se asocia a la baja reacción de generación de las moléculas oxidadas seguida por la inyección del $\mathrm{ZnO}$, causado generalmente por la no-exposición a la luz, debido al enmascaramiento que genera el electrolito. Este fenómeno de-exposición a la luz genera una concentración de estado estacionario del colorante oxidado, sugiriendo la presencia de una interfaz de carga positiva, un incremento de la recombinación de portadores de carga generando como resultado una respuesta de fotocorriente baja. Cuando se deja de generar luz, la recombinación se hace presente en forma de un pequeño pico catódico, sugiriendo una descarga de la carga superficial positiva por el electrodo ${ }^{[13]}$.

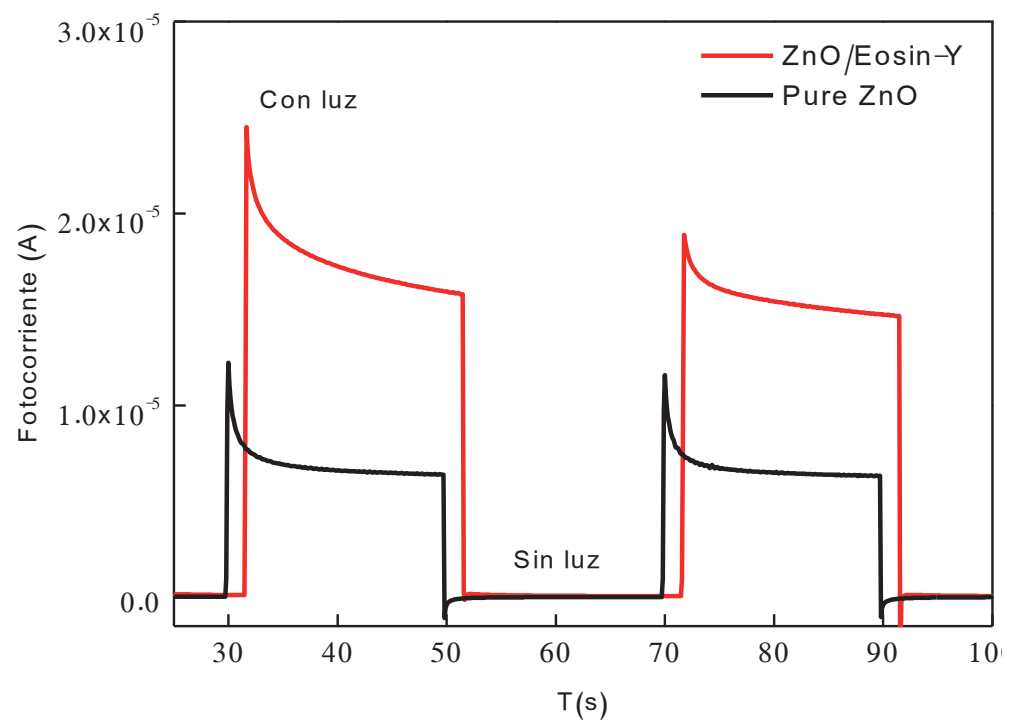

Figura 5. Medidas de fotocorriente para las películas de $\mathrm{ZnO}$ puro y las películas híbridas de $\mathrm{ZnO}$ /Eosin-Y.

\section{Conclusiones}

Se comprobó que la electrodeposición catódica es un método confiable y eficiente para la obtención de materiales nanoestructurados. Fueron estudiados los cambios en la morfología de las películas de $\mathrm{ZnO}$ mediante el uso de Eosin-Y como colorante durante la síntesis. Se encontró una morfología altamente porosa cuando es utilizada una concentración de [Eosin-Y] $=1 \times 10^{-4} \mathrm{M}$, haciéndola apta para la EDC de materiales híbridos. Además, que la calidad cristalina aumenta al utilizar colorantes en la disolución, mientras que la orientación del $\mathrm{ZnO}$ se mantiene en su dirección preferencial (002). La respuesta de fotocorriente indica que una alta proporción de las moléculas del colorante son excitadas por los electrones que actúan como sensibilizador. Podemos concluir que el uso de Eosin-Y como colorante puede ser utilizado para la obtención de materiales híbridos $\mathrm{ZnO} /$ Eosin-Y con el propósito de mejorar la eficiencia para aplicaciones en DSSC. 


\section{Referencias}

[1] B. O'Redan, M. Grätzel. Nature. 353, 737, 1991.

[2] W. J. Lee, H. Okada, A. Wakahara, A. Yoshida. Cream. International. 32, 495, 2006.

[3] M. Law, L. E. Greene, J. C. Johnson, >R. Saykally, P. Yang. Nature Materials. 4, 455, 2005.

[4] A. Hagfeldt, M. Grätzel. Chemical Reviews. 95, 1, 49, 1995.

[5] M. Grätzel. Nature. 414, 338, 2001.

[6] Th. Pauporte, A. Yoshida, A. Goux, D. Lincot. Journal of Electroanalytical Chemistry, 534, 55, 2002.

[7] C. Klingshirn. Phys. Status Solidi B71, 547, 1975.

[8] T. Yoshida, H. Minoura. Adv Mater. 12, 1219, 2000.

[9] F. Labat, I. Coifini, H. P. Hratchian, M. Frisch, K. Raghavachari, C. Adamo. JACS. 131, 14290, 2009.

[10] S. Junaid, S. Qazi, AR Rennie, JK Cockcroft, M. Vickers. J Colloid Interface Sci. 338, 105, 2009.

[11] Particle Size and Strain Analysis by X-Ray Diffraction: H \& M Analytical Services, Inc; 20002 [http://www.h-and-m-analytical. $\mathrm{com} / \mathrm{pdfs} /$ size_strain.pdf].

[12] T. Yoshida, M. Iwaya, H. Ando, T. Oekermann, K. Schlettwein, D. Wöhrle, H. Minoura. Chem Commun. 400, 2004.

[13] B. Marí, MM. Moya, KC. Singh, M. Mollar, P. Palacios, E. Artacho, P. Wahnón. J Electrochemical Chem. 653, 86, 2011. 